\title{
Excessive expression of miR-27 impairs Treg-mediated immunological tolerance
}

\author{
Leilani O. Cruz, ${ }^{1}$ Somaye Sadat Hashemifar, ${ }^{2}$ Cheng-Jang Wu, ${ }^{1}$ Sunglim Cho, ${ }^{1}$ Duc T. Nguyen, ${ }^{1}$ Ling-Li Lin, ${ }^{1}$ \\ Aly Azeem Khan, ${ }^{2}$ and Li-Fan Lu1,3,4 \\ 'Division of Biological Sciences, UCSD, La Jolla, California, USA. ${ }^{\top}$ Toyota Technological Institute at Chicago, Chicago, Illinois, USA. ${ }^{3}$ Moores Cancer Center and ${ }^{4}$ Center for Microbiome Innovation, \\ UCSD, La Jolla, California, USA.
}

\begin{abstract}
MicroRNAs (miRs) are tightly regulated in the immune system, and aberrant expression of miRs often results in hematopoietic malignancies and autoimmune diseases. Previously, it was suggested that elevated levels of miR-27 in T cells isolated from patients with multiple sclerosis facilitate disease progression by inhibiting Th2 immunity and promoting pathogenic Th1 responses. Here we have demonstrated that, although mice with T cell-specific overexpression of miR-27 harbor dysregulated Th1 responses and develop autoimmune pathology, these disease phenotypes are not driven by miR-27 in effector T cells in a cell-autonomous manner. Rather, dysregulation of Th1 responses and autoimmunity resulted from a perturbed Treg compartment. Excessive miR-27 expression in murine T cells severely impaired Treg differentiation. Moreover, Tregs with exaggerated miR-27-mediated gene regulation exhibited diminished homeostasis and suppressor function in vivo. Mechanistically, we determined that miR-27 represses several known as well as previously uncharacterized targets that play critical roles in controlling multiple aspects of Treg biology. Collectively, our data show that miR-27 functions as a key regulator in Treg development and function and suggest that proper regulation of miR-27 is pivotal to safeguarding Tregmediated immunological tolerance.
\end{abstract}

\section{Introduction}

MicroRNA-mediated (miR-mediated) gene regulation plays crucial roles in the development and function of the immune system $(1,2)$. The onset of many human hematopoietic malignancies can be directly attributed to the deletion or amplification of genomic regions where miRs are encoded (3-5). Similarly, abnormal expression of miRs in immune cells was also found to be highly correlated with the pathogenesis of a wide range of autoimmune disorders $(2,6)$. Accumulating experimental evidence has further demonstrated that certain miRs promote disease progression, whereas others function as negative regulators to limit immune activation and resultant pathologies (7-10). The identification of such a causative role of miRs in autoimmune disease pathogenesis suggests that miRs can serve as biomarkers and potential therapeutic targets for treating immunological disorders. Nevertheless, the precise molecular and cellular mechanisms by which miRs regulate autoimmunity require further investigation before miRbased immunotherapy is possible.

Previously, miR-27, a member of the miR-23 27 24 family, was found to be highly upregulated in T cells isolated from patients with multiple sclerosis (MS) (11). It was shown that through repressing BMI1, a molecule that stabilizes GATA3, miR-27 inhibits Th2 differentiation and promotes proinflammatory Th1 autoimmune responses. Consistent with this study, we have also recently found

Conflict of interest: The authors have declared that no conflict of interest exists. Submitted: May 6, 2016; Accepted: November 10, 2016.

Reference information: J Clin Invest. 2017;127(2):530-542.

https://doi.org/10.1172/JCI88415 that GATA3 itself can be directly targeted by miR-27, substantiating the idea that miR-27 is a negative regulator of Th2 immunity (12). Moreover, our recent finding of elevated IFN- $\gamma$ responses and lympho-hyperactivated phenotypes in mice harboring $\mathrm{T}$ cells with miR-27 overexpression further supported the conclusion drawn by the aforementioned study in patients with MS $(11,12)$. Surprisingly, the role of miR-27 in driving IFN- $\gamma$-mediated Th1 autoimmune responses implicated by these 2 reports seems to be at odds with previous studies, in which miR-27 was shown to be a potent repressor of IFN- $\gamma$ and $\mathrm{T}$ cell receptor-mediated (TCR-mediated) activation $(13,14)$. The fact that overexpression of miR-27 in T cells resulted in impaired Th1 differentiation further suggested that miR-27-mediated gene regulation limits, rather than promotes, Th1 responses (12). Such contradictory results warrant a more careful examination of the exact role of miR-27 in controlling $\mathrm{T}$ cell immunity.

In the current study, we show that miR-27 plays a cell-intrinsic role in negatively regulating effector $\mathrm{T}$ cell (Teff) homeostasis, activation, and cytokine production. Our results suggest that the dysregulated $\mathrm{T}$ cell responses and autoimmune phenotypes in mice with T cell-specific miR-27 overexpression are, on the other hand, likely due to a perturbed Treg compartment. A global transcription study, combined with high-throughput sequencing of RNAs isolated by cross-linking immunoprecipitation (HITS-CLIP) analysis, revealed that many genes previously associated with Treg differentiation and suppressor function are directly targeted by miR-27. In particular, we found that forced expression of miR-27 during thymocyte development severely impaired Treg generation by repressing c-Rel, a member of the NF- $\mathrm{BB}$ transcription factor family that is known for its indispensable role in initiating FOXP3 
A
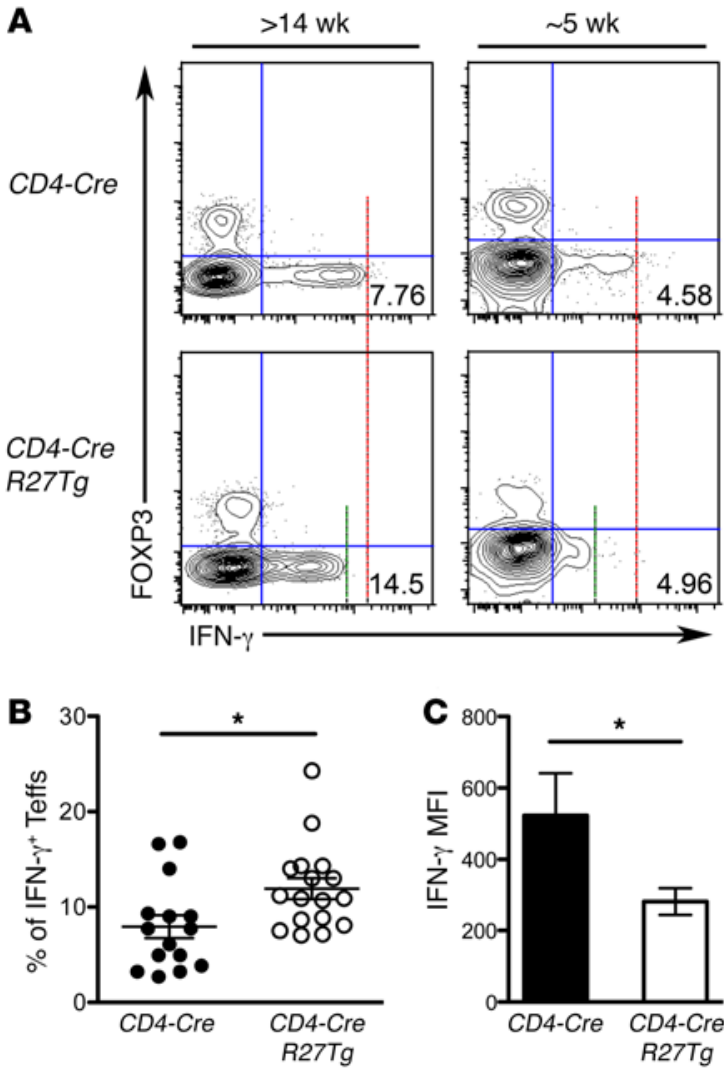

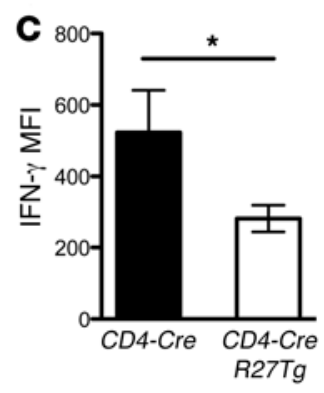

transcription (15-18). Exaggerated miR-27-mediated regulation of other Treg-suppressor molecules such as IL-10 and granzyme B (GZMB) further contributes to the functional defects in peripheral Treg populations, as miR-27-overexpressing Tregs failed to prevent autoimmune pathologies in the adoptive transfer colitis model as well as in mice with normal thymic Treg development. Together, our data provide new cellular and molecular insights into miR-27driven $\mathrm{T}$ cell autoimmunity and suggest that optimal expression of miR-27 is crucial for maintaining immunological tolerance through the regulation of multiple aspects of Treg biology.

\section{Results}

Mice harboring $T$ cells with miR-27 overexpression had dysregulated $I F N-\gamma$ responses despite diminished IFN- $\gamma$ production by $T$ cells on a per-cell basis. As previously reported, $\mathrm{T}$ cells in peripheral blood mononuclear cells (PBMCs) isolated from patients with MS had very high levels (up to 30 -fold induction) of miR-27 compared with those from healthy donors (11). To determine the precise role of miR-27 in regulating IFN- $\gamma$-dependent Th1 responses, we sought to further characterize mice harboring $\mathrm{T}$ cells that selectively overexpress miR-27 in CD4 cells (CD4-Cre R27Tg mice) at elevated levels similar to T cell levels found in MS patients (12). Previously, we showed that the spontaneous lympho-hyperactivation diseases that developed in mice with $\mathrm{T}$ cell-specific overexpression of the miR-23 27 24 family (CD4-Cre R23cTg) could be mostly recapitulated when miR-27 alone was overexpressed (12). Further analysis of mice of different ages revealed that the $\mathrm{T}$ cell-activation phenotypes could already be observed in approximately 3-monthold CD4-Cre R27Tg mice (Supplemental Figure 1A; supplemental

Figure 1. Diminished IFN- $\gamma$ production by miR-27-overexpressing $\mathrm{T}$ cells in CD4-Cre R27Tg mice. (A) FACS analysis of IFN- $\gamma^{+}$cells in FOXP3-CD4 ${ }^{+}$ Tconvs from mice of different ages ( $>14$ wk vs. $\sim 5 \mathrm{wk}$ ). (B) Frequencies and (C) mean fluorescence intensity (MFI) of IFN- $\gamma$ in R27Tg Tconvs compared with WT Tconvs from mice older than 14 weeks. Data represent the mean $\pm \mathrm{SD}$ and are representative of 3 independent experiments. Each symbol represents an individual mouse, and the bar represents the mean.

${ }^{*} P<0.05$. $P$ values are determined by an unpaired, 2-tailed Student's $t$ test.

material available online with this article; https://doi.org/10.1172/ JCI88415DS1). Five months after birth, these mice started to develop autoimmune disorders that manifested in runting, failure to thrive, lymphadenopathy and splenomegaly, and lymphocytic tissue infiltration that was particularly severe in the lung, colon, and stomach (Supplemental Figure 1B and data not shown). Interestingly, regardless of the absence or presence of increased IFN- $\gamma^{+} \mathrm{T}$ cell frequencies in younger or older CD4-Cre R27Tg mice, respectively (Figure 1, A and B), we could consistently detect reduced IFN- $\gamma$ production by miR-27-overexpressing $\mathrm{T}$ cells on a per-cell basis (Figure 1C), supporting the previous notion that miR-27 functions as a potent repressor of IFN- $\gamma$ in T cells $(13,14)$.

Enforced expression of miR-27 in $T$ cells results in reduced Treg numbers. The observation of dysregulated IFN- $\gamma$ responses in CD4-Cre R27Tg mice that harbored $\mathrm{T}$ cells with a diminished capacity to produce IFN- $\gamma$ raised the question as to whether the autoimmune pathology developed from a defect in the Treg compartment. After all, miR-27 was also overexpressed in Tregs in mice in which the Mir27 Tg was expressed in all T cells. To examine this possibility, we analyzed young CD4-Cre $R 27 \mathrm{Tg}$ mice $(<3$ months of age) to avoid the potential confounding effects from the aforementioned late-developing lympho-hyperactivation phenotypes. Indeed, while young mice did not show any clear alteration in total thymic or splenic cellularity compared with their WT littermates, we could easily detect a significant reduction in both thymic and splenic Treg populations (Figure 2, A-G). The observed Treg phenotype in CD4-Cre $\mathrm{R} 27 \mathrm{Tg}$ mice was mostly consistent with our previous findings in mice with $\mathrm{T}$ cell-specific overexpression of the entire miR-23 27 24 family (12), with an even greater percentage of the former showing decreased peripheral Treg frequencies (Figure $2 \mathrm{H}$ ). In contrast, some mice with $\mathrm{T}$ cell-specific overexpression of miR-24 (CD4-Cre R24Tg mice) actually harbored increased numbers of thymic Tregs, while no change in thymic or peripheral Treg frequencies could be detected in mice containing miR-23-overexpressing $\mathrm{T}$ cells (CD4-Cre R23Tg mice) (Figure 2H). Given that overexpression of miR-27 was also previously shown to strongly impair TGF- $\beta$-mediated induced Treg (iTreg) differentiation $(12,19)$, these results suggested that miR-27 is the major member of the miR-23 27 24 family that negatively regulates the Treg population.

miR-27 inhibits Teff homeostasis and function in a cell-autonomous manner. While insufficient numbers of Tregs probably contributed to the lympho-hyperactivation phenotypes observed in CD4-Cre $R 27 \mathrm{Tg}$ mice, the possibility that overexpression of miR-27 might lead to dysregulated $\mathrm{T}$ cell responses in a conventional $\mathrm{T}$ cell-intrinsic (Tconv-intrinsic) manner still cannot be fully excluded. To directly address this issue, we performed BM chimera studies by transferring BM cells from CD4-Cre R27Tg mice or WT littermates mixed 
A
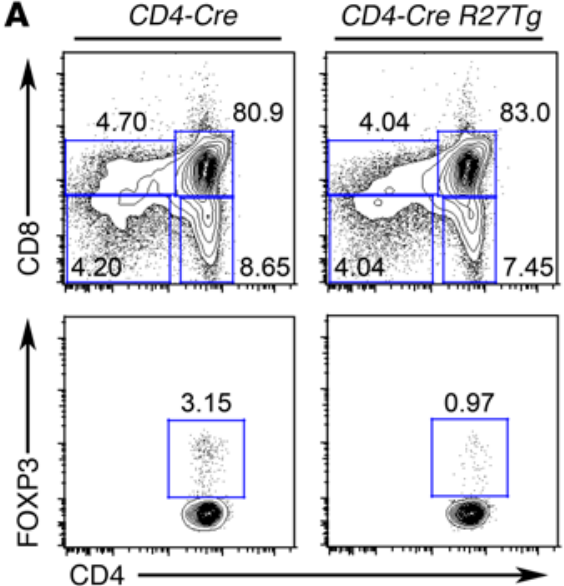
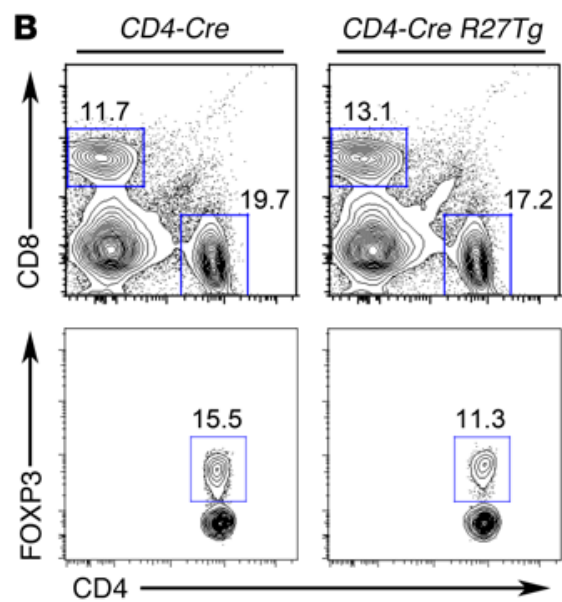
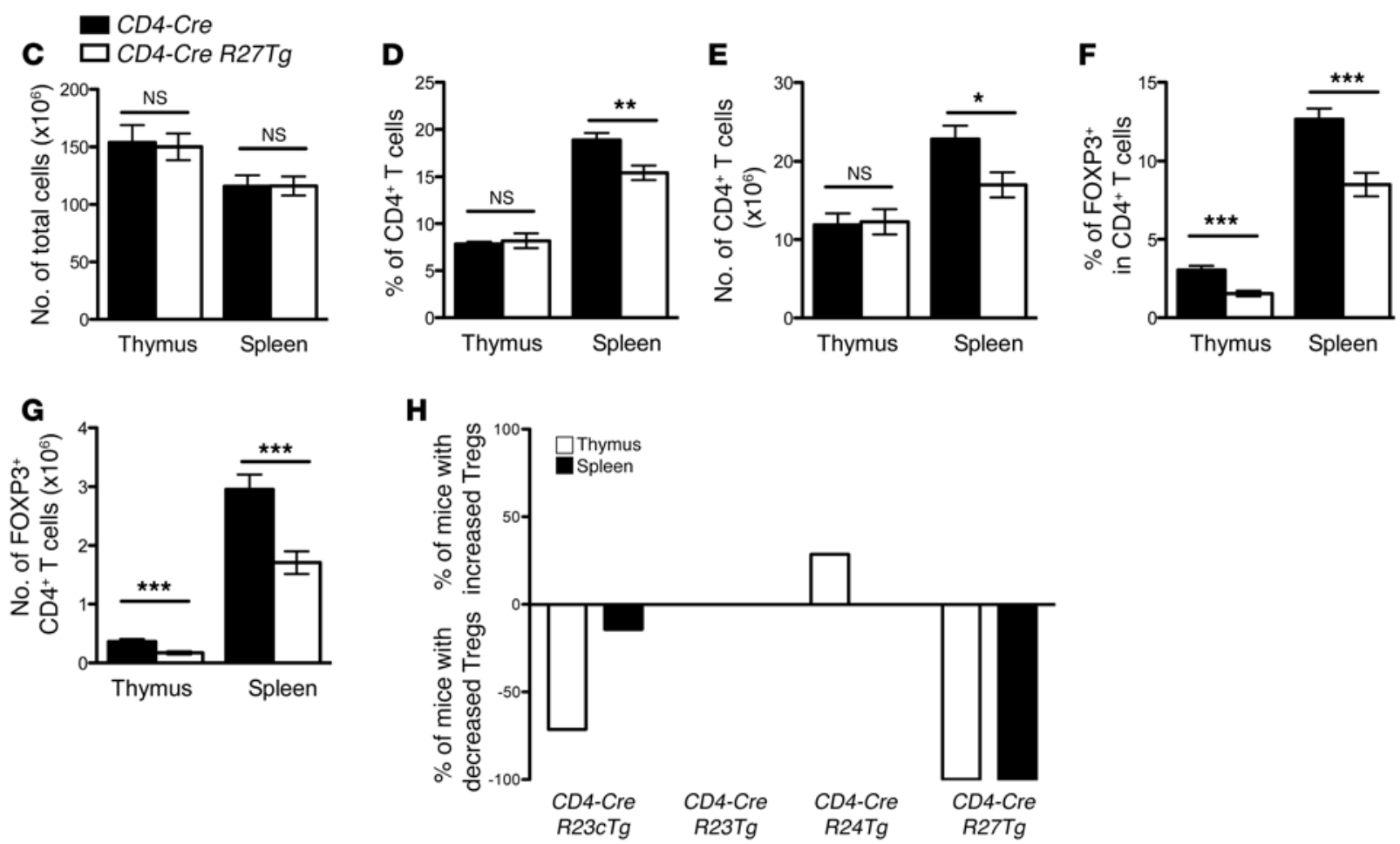

Figure 2. CD4-Cre R27Tg mice harbor reduced Treg numbers. FACS analysis of (A) thymus and (B) spleen of 6- to 12-week-old CD4-Cre R27Tg mice and their WT littermates. (C-C) Cellularity of the thymus and spleen and the proportions and absolute numbers of thymic and splenic total CD4+ $T$ cells and FOXP3+CD4+ Tregs in CD4-Cre R27Tg mice and their control littermates. (H) Percentage of mice with overexpression of the whole miR-23 cluster, or individual members, showed increased or decreased Treg frequencies in thymus and spleen compared with their corresponding WT littermates. Data represent the mean \pm SD and are representative of 3 independent experiments $(n=6-17) .{ }^{*} P<0.05,{ }^{* *} P<0.01$, and ${ }^{* * *} P<0.001$. $P$ values are determined by an unpaired, 2-tailed Student's $t$ test.

with BM cells from Ly5.1+ $\mathrm{B} 6$ mice at a 1:1 ratio into irradiated Rag1deficient mice, as described previously (20) (Figure 3A). Unlike CD4-Cre R27Tg mice, the CD4-Cre R27Tg Ly5.1+ $\mathrm{B} 6$ chimeric mice were healthy and showed no sign of autoimmunity and no clear alterations in different thymocyte subsets (Supplemental Figure 2A). Nevertheless, consistent with our observation in unmanipulated CD4-Cre R27Tg mice, we could easily detect marked reductions in the frequencies of R27Tg Tregs within both the thymic and peripheral $\mathrm{CD} 4^{+} \mathrm{T}$ cell populations as compared with the corresponding WT cell subsets in the control mixed chimeras (Figure 3B). Moreover, R27Tg Tregs also had a reduced proliferative capacity as shown by reduced Ki67 staining (Supplemental Figure 3), indicating a cellintrinsic role of miR-27 in controlling Treg homeostasis. On the other hand, contrary to what has been shown in CD4-Cre R27Tg mice, we observed reduced $\mathrm{R} 27 \mathrm{Tg} \mathrm{CD} 4^{+} \mathrm{T}$ cell frequencies in the periphery, with diminished proliferation, reduced activation, and impaired cytokine production compared with their WT counterparts (Figure 3, C-E and Supplemental Figure 2B). These results implied that the cell-autonomous role of miR-27 in Teffs is that of inhibiting rather than promoting homeostasis and effector function and strongly suggested that a perturbed Treg compartment in CD4-Cre R27Tg mice is responsible for the observed autoimmune pathology. 
A

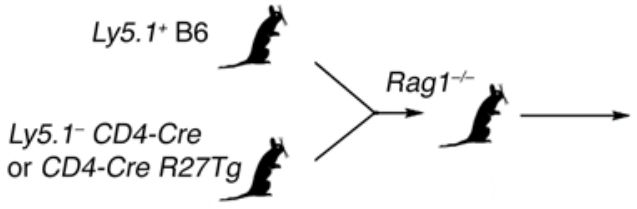

B
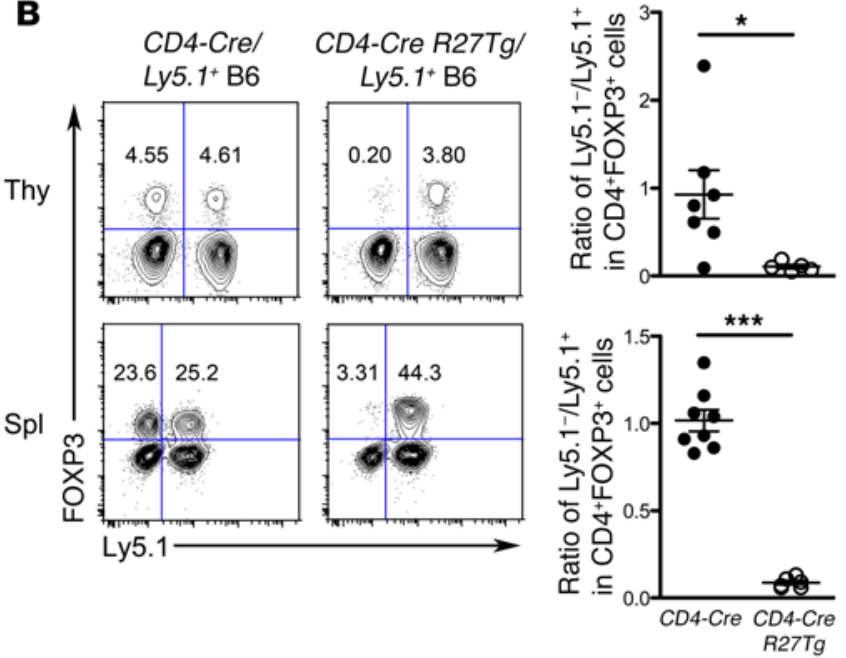

C


D
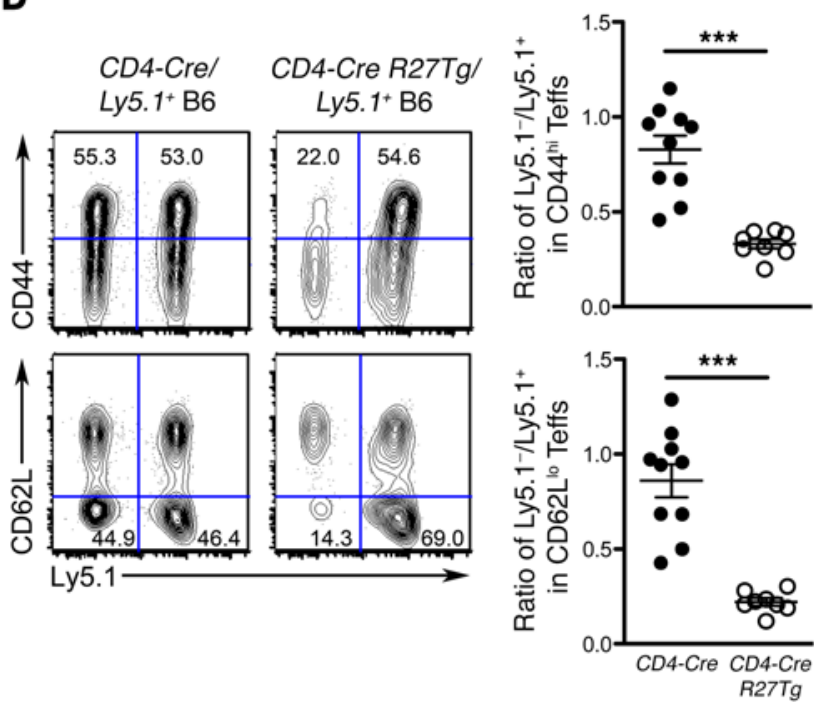

$\mathbf{E}$

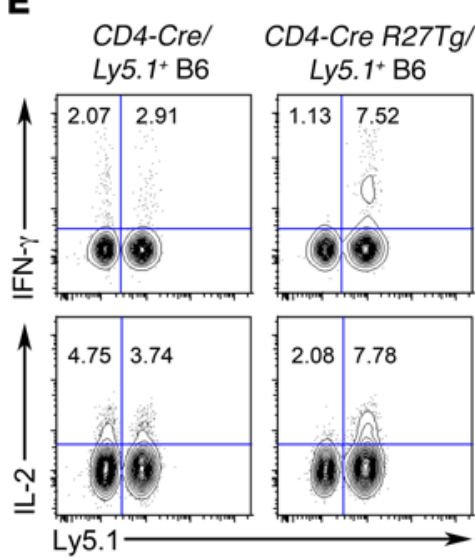

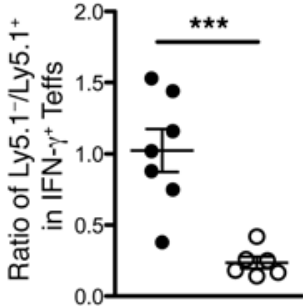

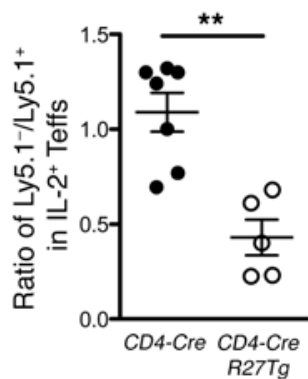

Figure 3. Excessive miR-27 expression impairs Teff activation and function. (A) Schematic of the generation of mixed BM chimeric mice. (B) FACS analysis and ratios of the frequencies of Ly5.1 ${ }^{-} \mathrm{FOXP3}{ }^{+}$and Ly5.1 ${ }^{+} \mathrm{FOXP3}{ }^{+}$cells within each donor-derived total CD4 ${ }^{+} \mathrm{T}$ cell population from both thymus (Thy) and


CD62 $\mathrm{L}^{10}$, as well as (E) Ly5.1-IFN- $\gamma^{+}$and Ly5.1 $1^{+} \mathrm{IFN}-\gamma^{+}$and Ly5.1-IL-2 $2^{+}$and Ly5.1+IL-2+ splenic Tconvs. FACS data represent 3 independent experiments. Each symbol represents an individual mouse, and the bar represents the mean. ${ }^{*} P<0.05,{ }^{* *} P<0.01$, and ${ }^{* * *} P<0.001$. $P$ values are determined by an unpaired, 2-tailed Student's $t$ test.

Excessive miR-27 expression impairs both thymic Treg development and peripheral Treg homeostasis. Given our observation of reduced thymic Treg numbers in CD4-Cre R27Tg mice, we hypothesized that the expression of miR-27 needs to be tightly regulated and that downregulation of miR-27 prior to FOXP3 expression is required to ensure normal Treg development. Indeed, among all thymocyte populations, we found that miR-27a (and miR-27b to a lesser degree) was expressed at the lowest level in the FOXP3- CD4SP subset (Figure 4A). On the other hand, the expression level of miR24, another member of the miR-23 27 24 family that seemed to promote rather than inhibit thymic Treg development upon overexpression (Figure 2C), did not change during the transition from double-positive (DP) to CD4 single-positive (CD4SP) cell stages (Supplemental Figure 4). Nonetheless, it remains possible that
miR-27 inhibits only Treg homeostasis similarly to how it regulates the Teffs described above. To address this, we generated heterozygous Foxp $3^{\mathrm{Cre} / \mathrm{+}} \mathrm{R} 27 \mathrm{Tg}$ female mice, which harbor a Treg population with miR-27 overexpression driven by FOXP3 and a WT Treg population caused by X chromosome inactivation (21). Moreover, as Cre recombinase is fused to a yellow fluorescent protein (YFP), Tregs subjected to Cre-mediated Tg expression can be detected on the basis of the expression of YFP (22). Like the mixed BM chimeras, Foxp $3^{\mathrm{Cre} / \mathrm{+}} \mathrm{R} 27 \mathrm{Tg}$ mice did not develop any detectable immune phenotype, probably because of the presence of WT Tregs. Interestingly, when expression of the Mir27 Tg was induced strictly after thymic Tregs were differentiated (i.e., expression of FOXP3), we could only detect reduced proportions of FOXP3-driven YFP-Cre ${ }^{+}$ miR-27-overexpressing Tregs in the spleen and peripheral tissues, 
A
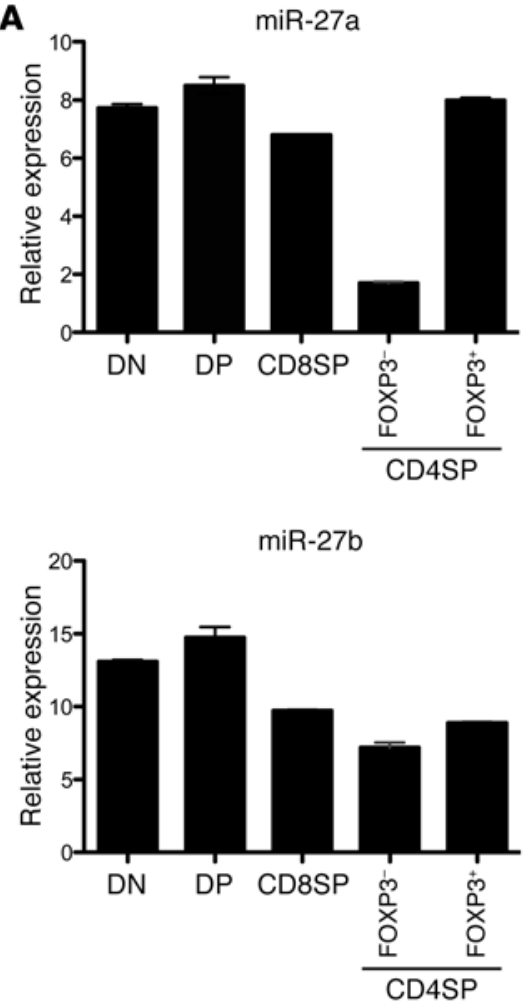

B

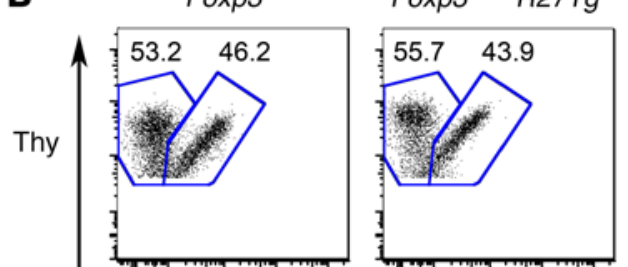

Spl

LP

芫
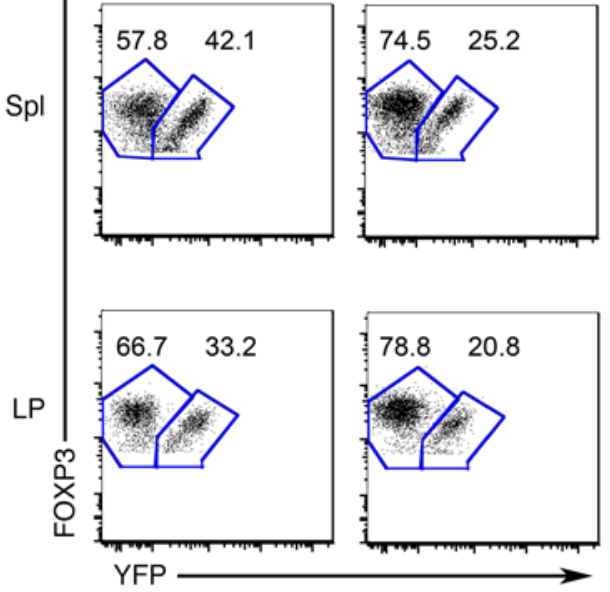

C

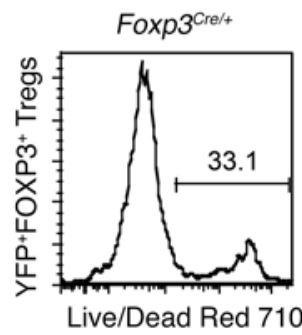

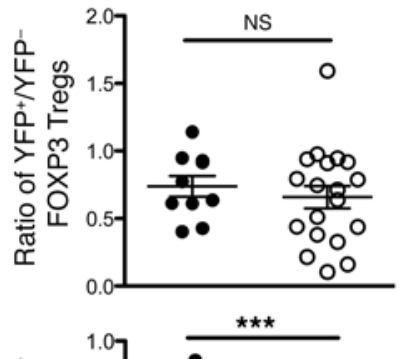
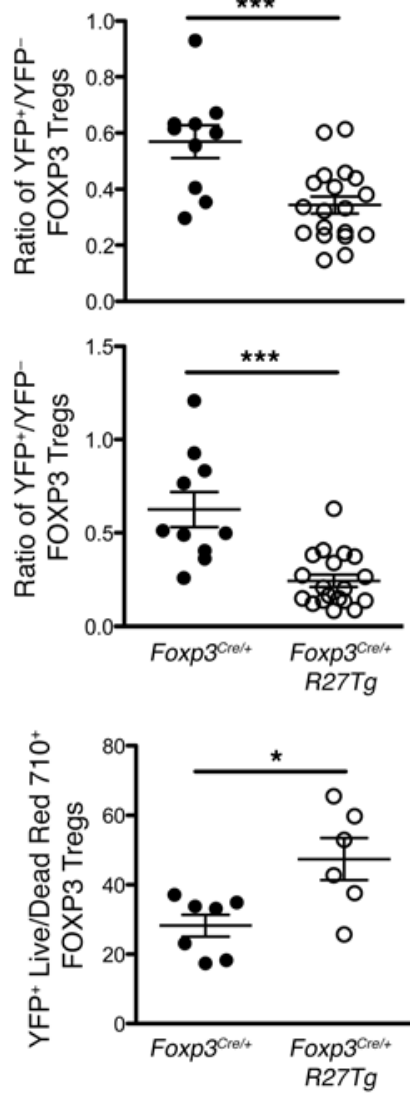

Figure 4. Optimal miR-27 expression is required to maintain normal Treg development and homeostasis. (A) $q P C R$ analysis of miR-27a/b expression in different thymocyte subsets. Data represent the mean \pm SD and are representative of 3 independent experiments $(n=3-6)$. (B) FACS analysis and ratios of YFP-Cre ${ }^{+}$and YFP-Cre ${ }^{-}$cells within the FOXP3 ${ }^{+} \mathrm{CD}^{+}{ }^{+}$cell population from FOXP3 ${ }^{\mathrm{Cre} /+} \mathrm{R} 27 \mathrm{Tg}$ mice and FOXP3 ${ }^{\mathrm{Cre} /+}$ control females. FACS data represent 4 independent experiments. (C) Viability of YFP-Cre+ Tregs from the indicated mice was measured by Live/Dead staining after in vitro stimulation with plate-bound anti-CD3 and anti-CD28 for 6 hours. FACS data represent 3 independent experiments. Each symbol represents an individual mouse, and the bar represents the mean. ${ }^{*} P<0.05$ and ${ }^{* * *} P<0.001$. $P$ values are determined by an unpaired, 2-tailed Student's $t$ test.

whereas the frequencies of thymic Tregs were comparable, regardless of the miR-27 expression levels (Figure 4B), suggesting that excessive miR-27 expression prior to FOXP3 induction perturbs Treg development in the thymus. Moreover, continued miR-27 overexpression after FOXP3 induction would further impair the peripheral Treg homeostasis that was observable in the aforementioned proliferation defects and impaired survival (Figure 4C). On the other hand, miR-27 seemed to play only a minimal role in controlling Treg tissue trafficking, as the frequencies of miR-27-overexpressing Tregs compared with those of their WT counterparts were similarly reduced across different tissues (Supplemental Figure 5A). Consistent with this finding, except for lower CD103 expression, we detected no reduction in expression of the corresponding chemokine receptors in Tregs with excessive miR-27 expression (Supplemental Figure 5, B and C).
miR-27 targets c-Rel, a member of the NF- $\kappa B$ transcription factors crucial for Treg differentiation and homeostasis. While most research efforts to date on miR-27 have been primarily focused on its role in tumorigenesis and embryonic stem cell differentiation (23-26), many miR-27 targets identified in those studies such as FOXO1, runt-related transcription factor 1 (RUNX1), and SMAD2/3 were known to regulate Treg biology as well (27-32). We found that, while FOXO1 did not seem to be repressed by miR-27 in T cells, markedly diminished SMAD2/3 and RUNX1 protein levels were detected in T cells that overexpressed miR-27 (Supplemental Figure 6). Impaired TGF- $\beta$ signaling resulting from miR-27-mediated repression of SMADs probably contributed to the impaired iTreg differentiation phenotype described in our previous study (12). On the other hand, despite a well-established role of the RUNX1CBF $\beta$ complex in maintaining FOXP3 expression $(30,33)$, exces- 
A

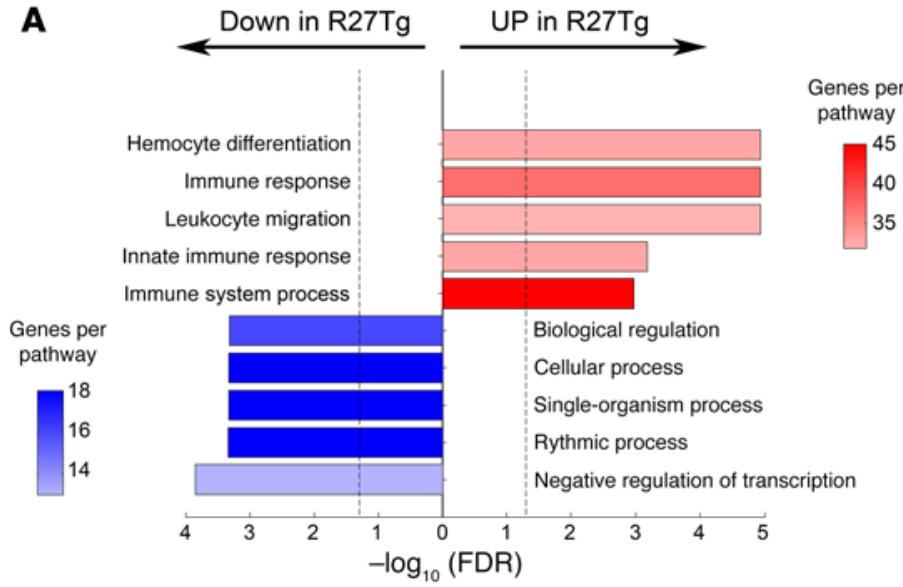

C

- All Treg genes

- Treg genes with positive HITS-CLIP hit(s)

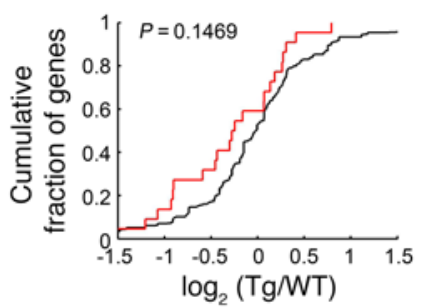

D

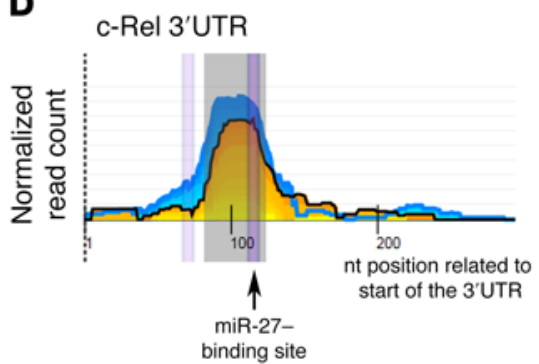

E
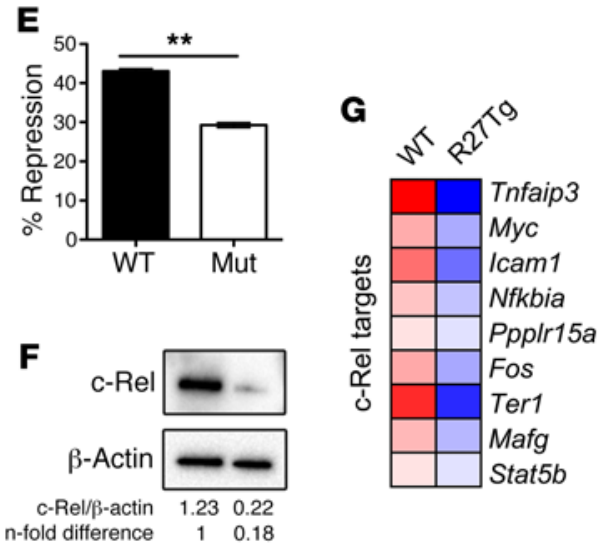

Figure 5. miR-27 targets c-Rel and controls other genes important for Treg differentiation and homeostasis. (A) Annotated CO biological processes were assigned to genes differentially expressed in naive T cells with or without miR-27 overexpression, as determined by RNA-seq. (B) Heatmap of representative genes associated with Treg differentiation that were differentially expressed in the presence of excessive miR-27 expression. (C) Cumulative distribution frequency plots depict the effect of overexpression of miR-27 on mRNA expression of Treg-associated genes. mRNA levels of Treg-associated genes bearing HITS-CLIP-identified miR-27 sites (red line) were compared with mRNA levels of Treg-associated genes (black line). (D) HITS-CLIP analysis of the putative miR-27 site in the 3'-UTR of c-Rel. (E) Ratios of repressed luciferase activity of cells in the presence of the c-Rel 3'-UTR with or without mutations (Mut) in the seed sequences in the presence of miR-27 compared with cells transfected with control miR. (F) Immunoblot analysis of c-Rel expression in T cells with or without miR-27 overexpression. Densitometric c-Rel expression values were normalized to $\beta$-actin expression values and n-fold increase on the basis of each corresponding WT sample. (G) Heatmap of representative c-Rel-targeted genes differentially expressed in the presence of excessive miR-27. Data represent the mean \pm SD and are representative of 3 independent experiments $(n=3-6)$. ${ }^{*} P<0.01$. $P$ values are determined by an unpaired, 2-tailed Student's $t$ test.

sive miR-27 expression only led to a marginal reduction in FOXP3 expression in the peripheral Tregs (Supplemental Figure 7), suggesting that the remaining RUNX1 protein level was sufficient to maintain FOXP3 expression in Tregs.

Neither SMAD2/3 nor RUNX1 has been clearly shown to regulate thymic Treg development or peripheral Treg homeostasis (34, 35). To gain further molecular insights into the mechanistic aspect of how miR-27 controls Treg differentiation and homeostasis, we first reanalyzed the recently reported RNA-sequencing (RNAseq) data on R27Tg T cells (12). Gene ontology (GO) enrichment analysis demonstrated that excessive miR-27 expression resulted in substantial changes in genes related to immune responses and hemocyte differentiation (Figure 5A). Additional screening of genes associated with Treg differentiation further revealed that Rel (the gene that encodes c-Rel), Nr4a1-3, and Foxo3, as well as the aforementioned miR-27 target genes Smad2 and Smad3, were downregulated in T cells with miR-27 overexpression (Figure $5 \mathrm{~B}$ ). c-Rel, NR4A proteins, and FOXO3 have all been shown to play crucial roles in regulating thymic Treg development (15-18, 27, $36,37)$. Moreover, even though miR-27 generally does not seem to repress Treg-related genes at the mRNA level, as demonstrated by our cumulative distribution frequency (CDF) plot analysis (Figure 5C), further analysis of the previously established high-throughput sequencing of RNAs isolated by HITS-CLIP results has identified c-Rel as a potential miR-27 target (Figure 5D) (38). Supporting this notion, our luciferase reporter studies confirmed that miR-27 can directly repress c-Rel (Figure 5E), and R27Tg T cells expressed significantly reduced amounts of c-Rel protein (Figure 5F). Consistently, many previously identified c-Rel targets were also downregulated in T cells with miR-27 overexpression (39) (Figure 5G). These findings strongly suggested that excessive expression of miR-27 could negatively impact FOXP3 induction and Treg development in the thymus through targeting of c-Rel (16). Moreover, compromised c-Rel-dependent gene regulation could further contribute to the impaired peripheral Treg homeostasis observed in mice harboring miR-27-overexpressing Tregs (15).

miR-27-overexpressing Tregs fail to control effector $T$ cell activation and function. Given the reduction in Treg numbers, we next sought to examine whether excessive miR-27 expression would also lead to impaired Treg function. Despite displaying a comparable in vitro suppression capacity (Supplemental Figure 8) and unlike WT Tregs, we found that cotransferred R27Tg Tregs failed to protect 
A

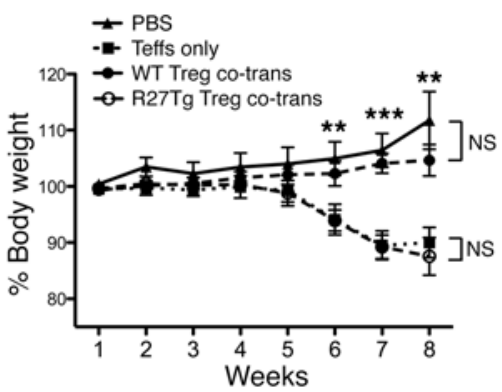

c

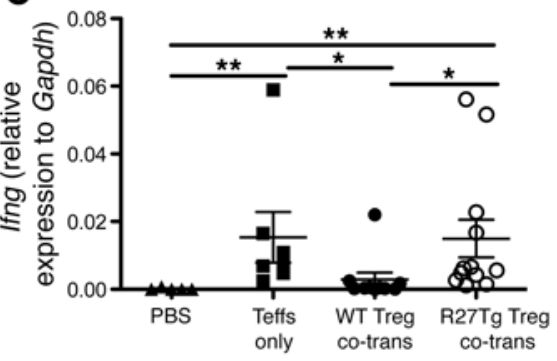

$\mathbf{F}$

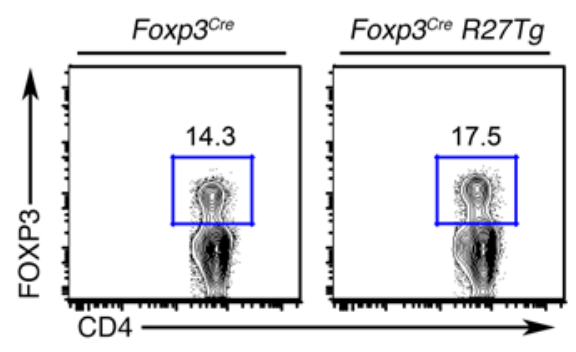

G

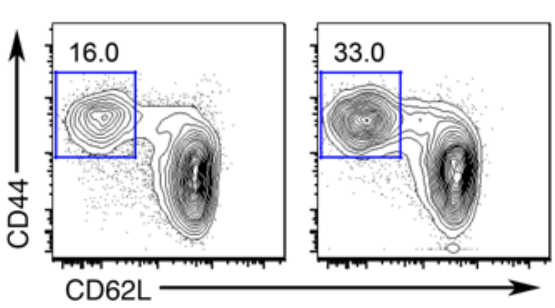

H
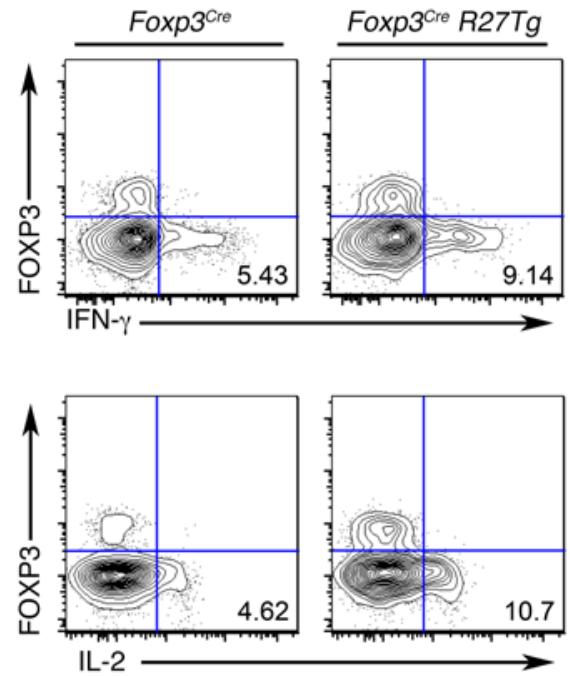

B
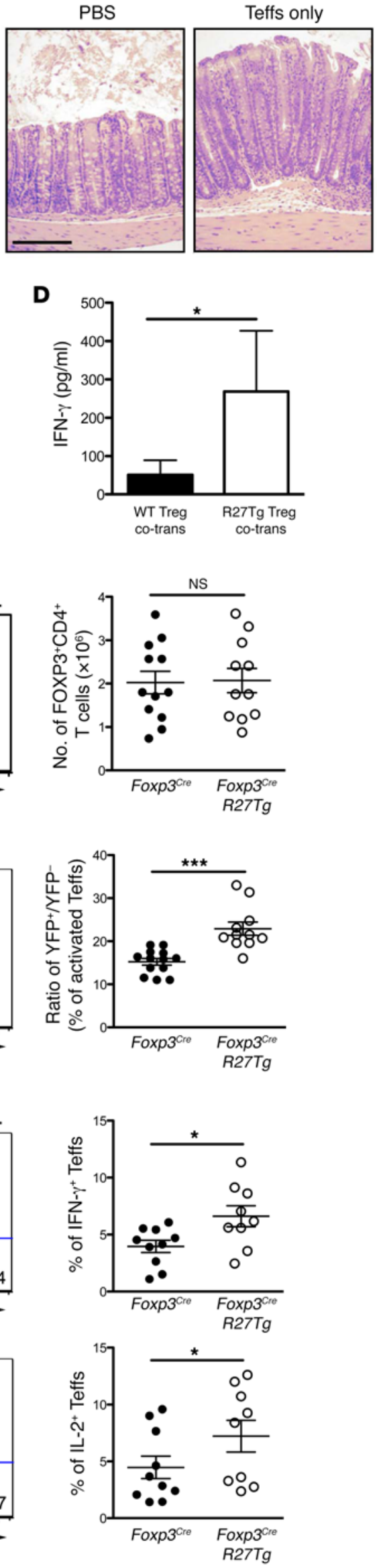

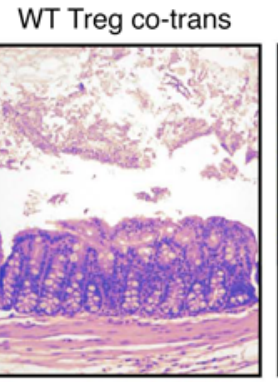

R27Tg Treg co-trans

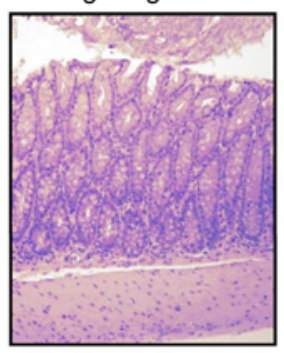

E
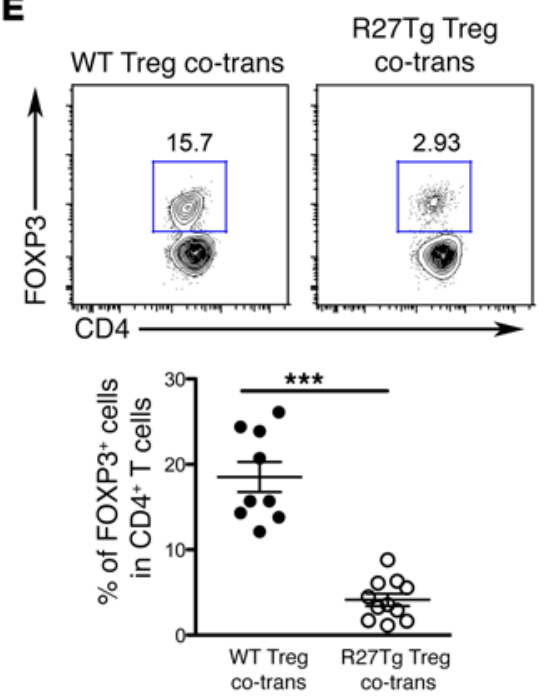

Figure 6. Tregs with miR-27 overexpression fail to maintain immunological tolerance. (A) Percentage of BW change of Rag T/ $^{-/}$recipient mice after adoptive transfer of $4 \times 10^{5} \mathrm{WT}\left(\mathrm{CD} 4^{+}\right.$ CD45RB ${ }^{\text {hiCD25-) }} \mathrm{T}$ cells with or without $2 \times 10^{5}$ WT or R27Tg Tregs (CD4+CD25 hi). PBS injection only was used as the no-T cell transfer control. (B) Eight weeks after adoptive transfer, colon sections from the mice were stained with H\&E for microscopic imaging. Scale bar: $50 \mu \mathrm{m}$. (C) qPCR and (D) ELISA of IFN- $\gamma$ mRNA and protein levels in colonic tissues harvested from the indicated mice. (E) FACS analysis and frequencies of $\mathrm{FOXP3}^{+}$ cells in total CD4 ${ }^{+} T$ cells isolated from LP. FACS analysis and frequencies of (F) FOXP3 ${ }^{+}$cells in total CD4 ${ }^{+} T$ cells, (G) CD44 $4^{\text {hi }}$ CD62 $\mathrm{L}^{10}$ cells, and (H) IFN $-\gamma^{+}$and IL-2+ cells in Tconvs from spleens of 6-week-old Foxp $3^{\text {cre }}$ R27Tg mice or WT controls. Data represent the mean $\pm \mathrm{SD}$ and are representative of 3 independent experiments. Each symbol represents an individual mouse, and the bar represents the mean. ${ }^{*} P<0.05$, ${ }^{*} P<0.01$, and ${ }^{*} *{ }^{*} P<0.001$. $P$ values in (C) are determined by one-way ANOVA and by an unpaired, 2-tailed Student's $t$ test in all others. co-trans, cotransfer. 
mice from weight loss and colonic pathology in the $\mathrm{CD} 4^{+} \mathrm{CD} 45 \mathrm{Rb}$ transfer model of colitis (40) (Figure 6, A and B). Moreover, we detected significantly higher IFN- $\gamma$ mRNA and protein levels in colons harvested from mice cotransferred with miR-27-overexpressing Tregs compared with mice that received WT Tregs (Figure 6, C and D). By contrast, transfer of R27Tg Tconvs alone did not result in weight loss or colitis (Supplemental Figure 9 and data not shown). These results not only confirmed the aforementioned negative role of miR-27 in Teff homeostasis and function, but also demonstrated that excessive miR-27 expression impaired Tregmediated regulation of Teff-driven intestinal autoimmunity. Nonetheless, it should be noted that mice receiving miR-27-overexpressing Tregs also had a significantly lower proportion of FOXP3 ${ }^{+}$ cells at 8 weeks after cotransfer, probably because of the aforementioned homeostatic defect (Figure 6E). Therefore, it remained unclear whether the uncontrolled intestinal pathology in mice cotransferred with miR-27-overexpressing Tregs was simply due to a lack of sufficient Treg numbers or the combination of reduced numbers and impaired suppressor function. To address this question, we used hemizygous Foxp $3^{\text {Cre }} R 27 \mathrm{Tg}$ male mice. At a young age, Foxp $3^{\text {Cre }} \mathrm{R} 27 \mathrm{Tg}$ mice harbored Treg numbers similar to numbers detected in their WT littermates (Figure 6F), allowing us to directly examine the impact of miR-27 overexpression on Treg-suppressor function in an in vivo setting. To this end, we could easily detect enhanced activation of splenic Teffs with an increased CD $44^{\text {hi }}$ CD62 $\mathrm{L}^{\text {lo }}$ cell subset and elevated production of IFN- $\gamma$ and IL-2 in Foxp $3^{\mathrm{Cre}} \mathrm{R} 27 \mathrm{Tg}$ mice compared with their WT control littermates (Figure 6, G and H). Likewise, while it is difficult to distinguish the difference in $\mathrm{T}$ cell activation in lamina propria (LP) between Foxp $3^{\text {Cre }} \mathrm{R} 27 \mathrm{Tg}$ mice and their WT littermates, as almost all LP T cells were already highly activated, elevated proliferation and increased production of inflammatory cytokines (e.g., IL-17) in T cells from LP could be easily detected, despite normal Treg numbers (Supplemental Figure 10). These results strongly suggested that excessive expression of miR-27 is detrimental to the suppressive capacity of Tregs to control Teff activation and function.

Exaggerated miR-27-mediated regulation inhibits Treg function through repression of both known and novel targets. To explore the potential molecular mechanisms underlying miR-27-mediated regulation of Treg-suppressor function, we first performed an unbiased transcriptomic analysis of Tregs that overexpressed miR-27 as well as other miR-23 family members. Similar to what was reported in Tconvs (12), miR-27-overexpressing Tregs again exhibited the most distinct global gene expression profile compared with other Tg Treg populations (Figure 7A). Moreover, while it is not unexpected that miR-27 could mediate its effects in a cell-type-specific manner similar to that observed in other miRs (20), there was actually a strong positive correlation $(r=0.6383)$ in genes that were differentially expressed in Tregs versus those expressed in Tconvs in the presence or absence of miR-27 overexpression (Figure 7B), suggesting that miR-27 could target many gene pathways shared by Tregs and Tconvs. The fact that excessive miR-27 expression negatively impacted the homeostasis of both Tregs and Tconvs, as demonstrated by our mixed BM chimeras study, further supported this notion. Nevertheless, to directly assess the cell-intrinsic impact of excessive miR-27 expression on Tregs without the potential environmental influences in CD4-
Cre $R 27 \mathrm{Tg}$ mice that would develop autoimmunity over time, we performed another transcriptomic analyses of miR-27-overexpressing Tregs isolated from the aforementioned heterozygous Foxp $3^{\text {Cre/ }+} R 27 \mathrm{Tg}$ female mice. To this end, GO enrichment analysis identified genes related to immune system processes that were mostly affected by excessive miR-27 expression in Tregs (Supplemental Figure 11). Among them, many genes that are critical for Treg-suppressor function were shown to be downregulated in Tregs upon miR-27 overexpression (Figure 7C), providing the molecular basis for the autoimmune phenotypes observed in mice harboring miR-27-overexpressing Tregs.

Next, by cross-analyzing our RNA-seq data and previous HITS-CLIP results, we sought to identify miR-27 target(s) that could contribute to the impaired suppressor function detected in Tregs with exaggerated miR-27-mediated regulation. Although miR-27 in Tregs, on the whole, does not seem to regulate its targets at the mRNA level (Figure 7D), HITS-CLIP analysis nevertheless revealed that IL-10 and GZMB, 2 well-established Treg-suppressor molecules $(22,41,42)$, could be directly inhibited by miR-27 (Figure 7, E and F). It has previously been reported that miR-27 can regulate macrophage-mediated inflammatory responses through targeting of IL-10 (43). Our luciferase reporter results further demonstrated that, like IL-10, GZMB can also be directly repressed by miR-27 (Figure 7G). Consistent with these findings, reduced frequencies of IL-10- and GZMB-expressing Tregs with diminished IL-10 and GZMB protein production on a per-cell basis could be easily detected in the miR-27-overexpressing Tregs compared with the WT counterpart Tregs in the same Foxp3 $3^{\text {Cre/+ }}$ $\mathrm{R} 27 \mathrm{Tg}$ mice and their WT littermates (Figure 7, $\mathrm{H}$ and I). We also obtained similar results when TGF- $\beta$-dependent iTregs were examined (Supplemental Figure 12). Together, our study identified known (i.e., IL-10) as well as previously uncharacterized (i.e., GZMB) miR-27 targets that could play critical roles in the miR-27mediated regulation of Treg-suppressor function.

\section{Discussion}

Aberrant expression of miRs has been linked to a broad range of human diseases including both solid and liquid cancers, as well as metabolic, neurological, and various immunological disorders $(6,44-48)$. However, while distinct miR expression patterns serve as valuable biomarkers for disease diagnosis and prognosis, miRbased therapy is still in its infancy. In addition to overcoming the major challenge of effective delivery of miR or anti-miR constructs as potential therapeutic agents, understanding the precise role of a particular miR under a given disease setting is equally important to ensuring a successful therapeutic outcome. To this end, it was recently reported that $\mathrm{T}$ cells from patients with $\mathrm{MS}$ exhibited high levels of miR-27 expression and that exaggerated miR-2-mediated gene regulation promoted proinflammatory IFN $-\gamma$ responses through repression of BMI1-dependent Th2 differentiation (11). In this study, by taking advantage of a mouse model in which $\mathrm{T}$ cells express elevated levels of miR-27 similar to the levels identified in patients, we have provided direct experimental evidence to reconcile the seeming discrepancy in early studies regarding the impact of miR-27 on IFN- $\gamma$-mediated Th1 responses and further identified a previously unappreciated role of miR-27 in regulating multifaceted Treg biology. 


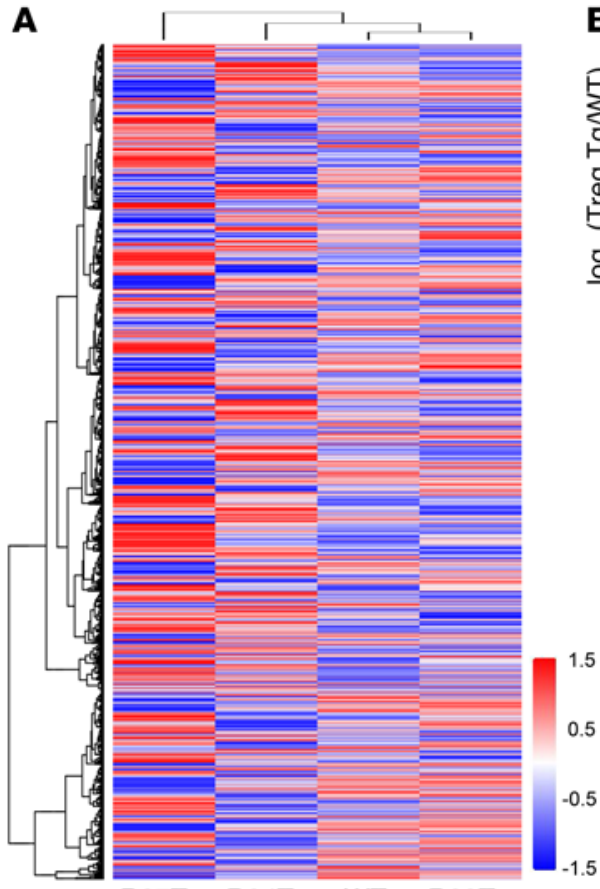

$\begin{array}{lll}\text { R27Tg R24Tg WT R23Tg } & \text { WT }\end{array}$
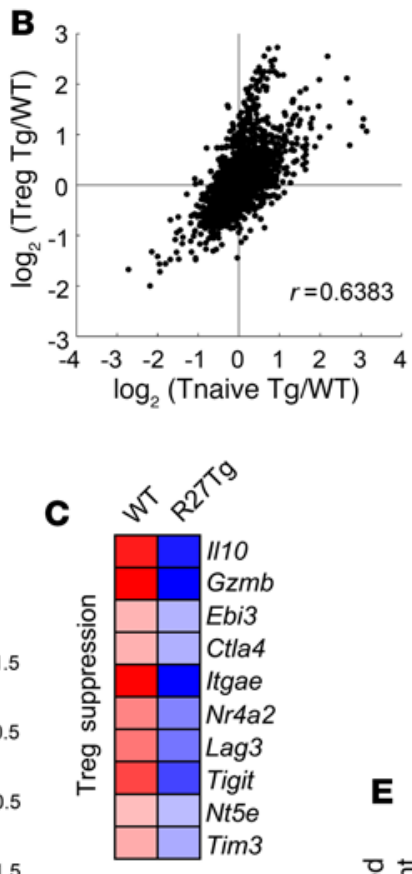

D - All genes
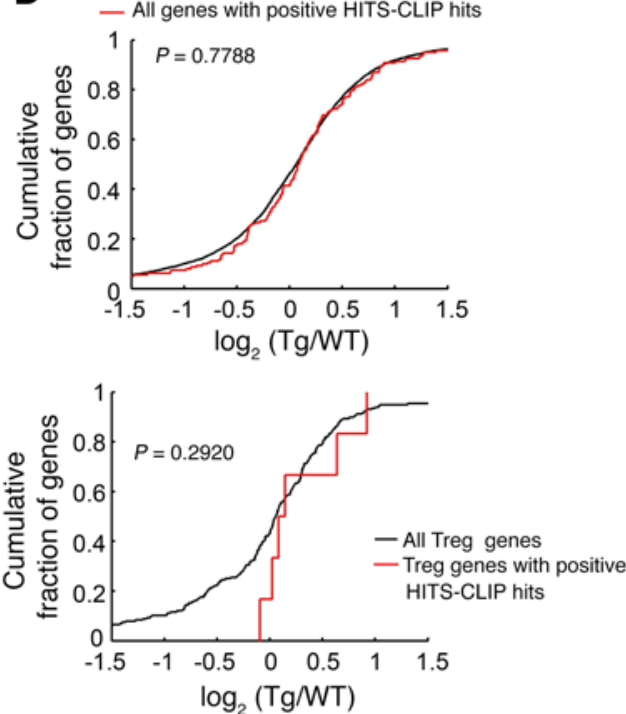

E IL-10 3'UTR

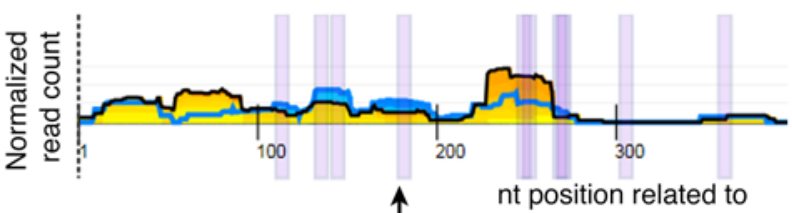

miR-27- start of the 3'UTR binding site

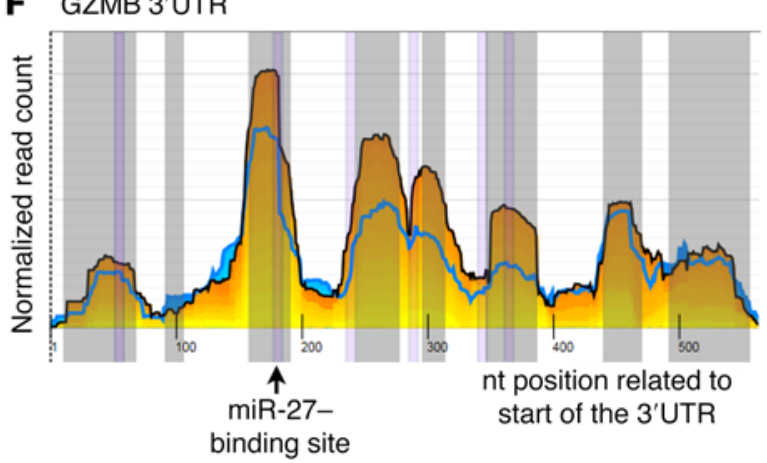

G

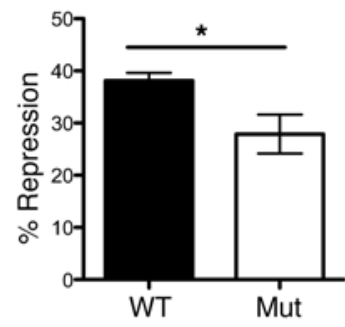

I
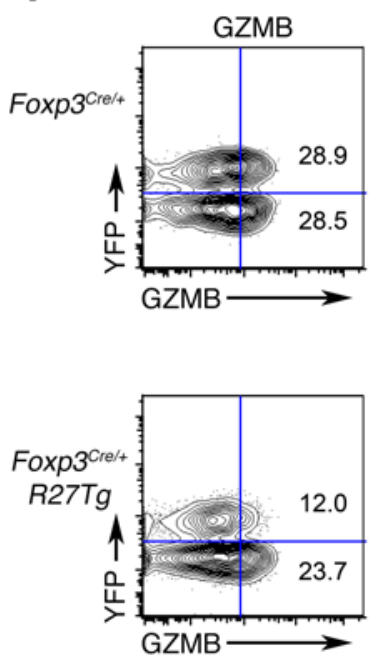
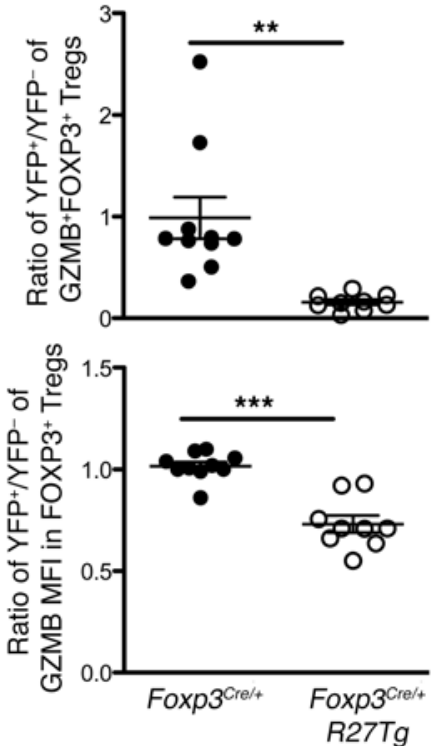
Figure 7. Multiple Treg-suppressor molecules including IL-10 and GZMB are regulated by miR-27. (A) Clustering of RNA-seq results from R23Tg, R24Tg, R27Tg, and WT CD4+CD25 hi Tregs based on total gene expression. (B) Scatter plot depicting the $\log _{2}$ fold changes of gene expression in naive R27Tg Tconvs over WT Tconvs versus R27Tg Tregs over WT Tregs. (C) Heatmap of the representative genes associated with Treg-suppressor function differentially expressed in YFP-Cre+ ${ }^{+}$Tregs isolated from either Foxp3 $3^{\mathrm{Cr} /+}$ R27Tg or Foxp $3^{\text {Cre/+ }}$ control mice. (D) CDF plots depicting the effect of overexpression of miR-27 on mRNA expression of Treg-associated or all genes. mRNA levels of Treg-associated or all genes bearing HITS-CLIP-identified miR-27 sites (red line) were compared with mRNA levels of total Tregassociated or all genes (black line). HITS-CLIP analysis of the putative miR27 site in the $3^{\prime}$-UTR of (E) IL-10 and (F) GZMB. Graph in $\mathbf{G}$ shows the ratios of repressed luciferase activity of cells in the presence of the GZMB $3^{\prime}$-UTR with or without mutations in the seed sequences in the presence of miR27 compared with cells transfected with control miR. FACS analysis, ratio of frequencies, and MFI of (H) IL-10 and (I) CZMB in LP FOXP3 ${ }^{+}$Tregs with (YFP-Cre ${ }^{+}$) or without (YFP-Cre-) miR-27 overexpression. Tregs were from Foxp $3^{\mathrm{Cre} /+} \mathrm{R} 27 \mathrm{Tg}$ and Foxp $3^{\mathrm{Cre} /+}$ control mice. Data represent the mean $\pm \mathrm{SD}$ and are representative of 3 independent experiments. Each symbol represents an individual mouse, and the bar represents the mean. ${ }^{*} P<0.05$, ${ }^{*} P<0.01$, and ${ }^{* *} P<0.001$. $P$ values are determined by an unpaired, 2-tailed Student's $t$ test.

Although miRs can certainly exert their biological function though the regulation of a single target (20), the effects of a given miR are usually subtle, and it is generally believed that miRs increase their impact by targeting a set of genes that are in a shared biological pathway (49). To this end, many known miR-27 targets such as FOXO1, RUNX1, SMAD2/3, and IL-10 have already been shown to play key roles in controlling different aspects of Treg biology. Even GATA3, a miR-27 target that contributes to miR-27dependent regulation of Th2 immunity (12), is also known for its role in maintaining Treg-suppressor function and identity (50-52). In the current study, we have further demonstrated that miR-27 can directly repress c-Rel and GZMB, 2 molecules that are important for Treg development, homeostasis, and suppressor function, respectively. While it is conceivable that there are more Treg-associated genes that can be regulated by miR-27 either directly or indirectly, we believe that these findings unequivocally identify miR-27 as one of the key molecular regulators in controlling Treg biology.

Considering the detrimental effects of excessive miR-27 expression on Treg homeostasis and function, it seemed counterintuitive that a miR normally expressed at higher levels in Tregs compared with their Tconv counterparts would play a negative role in controlling many aspects of the biology of these Tregs (12). However, this is actually not the first molecule prevalently expressed in Tregs that has been shown to be detrimental to the very same cell population when it is overexpressed. For example, while elevated T-bet expression is required for Tregs to limit Th1 inflammation (53), under extreme Th1-polarized conditions during Toxoplasma gondii infection or in the absence of cell-intrinsic negative regulators of the Th1 cytokine-signaling pathway, Tregs that acquired "supraoptimal" T-bet expression were shown to promote, rather than suppress, Th1 responses $(54,55)$. Moreover, this phenomenon is not only restricted to transcription factors in Tregs, but was also reported with miRs in other immune cell types. To this end, while miR-146a is generally considered an antiinflammatory miR, as mice with miR-146a ablation developed spontaneous autoimmune disorders (10), forced expression of miR-146a in mice also resulted in autoimmunity (56). It was suggested that different genes regulated by miR-146a were responsible for the seemingly contradictory phenotypes observed in $\mathrm{Tg}$ and KO mice. Thus, it is not surprising that miR-27 would also need to be properly controlled and that expression of miR-27 at either sub- or supraoptimal levels in Tregs could perturb their function. Indeed, while we were not able to delete only miR-27 in Tregs due to its genomic proximity to the other members in the miR-23 27 24 family, Tregs devoid of this miR family also showed impaired suppressor function. Consequently, mice with Treg-specific ablation of the miR23 27 24 family developed dysregulated IFN- $\gamma$ responses that were similar to what was observed in mice harboring Tregs with excessive miR-27 expression, further supporting the aforementioned scenario (C.J. Wu and L.F. Lu, unpublished results).

Our findings of impaired thymic Treg development in mice with $\mathrm{T}$ cell-specific miR-27 overexpression suggest that optimal miR-27 expression is not only required to safeguard Treg function in the periphery, but is also pivotal to maintaining normal Treg differentiation in the thymus. The fact that a marked drop in miR-27 levels occurs in FOXP3 CD4SP cells compared with DP thymocytes further supports the notion that miR-27 expressed at diminished levels immediately before Treg differentiation might be critical for the development of these Tregs in the thymus. Interestingly, among different members of the miR-23 27 24 family, only miR-27 plays a negative role in regulating thymic Treg development. While miR23 does not appear to be involved in this biological process, miR-24 might even promote Treg differentiation. Coincidently, unlike miR27, we detected no significant change in the expression of miR-24 in DP or FOXP3- CD4SP thymocytes. Therefore, while the entire miR-23 27 24 family is upregulated in FOXP3 $3^{+}$thymic Tregs, as was observed in the peripheral Tregs described previously (12), our results suggested that the expression of each miR-23 27 24 family member can be differentially regulated prior to FOXP3 induction to ensure proper Treg differentiation in the thymus.

Previously, it has been shown that miR-27 could attenuate T cell responses through direct targeting of $\operatorname{IFN}-\gamma(13,14)$. Therefore, it was initially puzzling to find dysregulated IFN- $\gamma$ responses in both patients with MS and mice harboring T cells with excessive miR-27 expression $(11,12)$. In addition to the negative impacts of miR-27 on TGF- $\beta$-mediated iTreg differentiation, as previously shown by us and others $(12,19)$, we demonstrated here that without directly impacting FOXP3 expression, exaggerated miR27-mediated gene regulation severely impaired Treg-mediated immunological tolerance and promoted T cell-mediated autoimmunity, even when Teffs themselves were impeded by miR-27 overexpression. Moreover, our experiments offer mechanistic insights into miR-27-mediated regulation of multifaceted Treg biology. We believe that our findings will facilitate the development of appropriate therapeutic strategies for the treatment of human immunological diseases.

\section{Methods}

Mice. CD4-Cre R23Tg, CD4-Cre R24Tg, CD4-Cre R27Tg, and CD4-Cre $R 23 c T g$ mice (12) as well as Foxp $3^{\text {Thyl.1 }}$ reporter mice (57) were described previously. $R 27 \mathrm{Tg}$ mice were bred with Foxp3 ${ }^{\mathrm{Cre}}$ mice (22) to obtain mice with Treg-specific overexpression of miR-27. Unless otherwise indicated, 6- to 12-week-old mice of both sexes were used. Neverthe- 
less, only WT littermates of the same sex served as controls in each individual experiment.

Flow cytometry. Single-cell suspensions of thymus, spleen as well as isolated LP lymphocytes were prepared as described previously (12). Cells were stained with Ghost Dye Red 780 (catalog 13-0865-T100; Tonbo Biosciences), followed by surface and intracellular antibody staining for CD4 (catalog 45-0042); CD8 (catalog 48-0081); CD44 (catalog 25-0441); CD62L (catalog 12-0621); CD103 (catalog 48-1031); CCR7 (catalog12-1971); CCR9 (catalog 25-1991); FOXP3 (catalog 53-5773); and Ki67 (catalog 51-5698) (all from eBioscience) at the manufacturer's recommended concentrations. Fixation and permeabilization of cells were performed with reagents from the Tonbo Biosciences FOXP3/ Transcription Factor Staining Kit (catalog TNB-0607). To detect IFN- $\gamma$ (catalog 17-7311); IL-17 (catalog 48-7177); and IL-2 (catalog 25-7021) (all from eBioscience) cytokine production, cells were stimulated in a 96-well plate with $50 \mathrm{ng} / \mathrm{ml} \mathrm{PMA}, 0.5 \mathrm{mg} / \mathrm{ml}$ ionomycin, and $1 \mathrm{mg} / \mathrm{ml}$ brefeldin A (all from Sigma-Aldrich) in complete 5\% RPMI media for 4 hours at $37^{\circ} \mathrm{C}$ before staining. To detect GZMB (catalog 50-8898; eBioscience) and IL-10 (catalog 12-7101; eBioscience) production, total splenocytes were stimulated with plate-bound $\alpha \mathrm{CD} 3$ (catalog BE0001; Bio X Cell) and $\alpha \mathrm{CD} 28$ (catalog BE0015; Bio X Cell), both at $1 \mu \mathrm{g} / \mathrm{ml}$, plus TGF- $\beta$ ( $5 \mathrm{ng} / \mathrm{ml})$ and IL-2 $(100 \mathrm{U} / \mathrm{ml})$ for 72 hours at $37^{\circ} \mathrm{C}$. Upon harvesting, cells were restimulated for 4 hours with PMA, ionomycin, and brefeldin A, as described above, before staining. To measure in vitro cell survival, total splenocytes from Foxp $3^{\mathrm{Cr} /+} \mathrm{R} 27 \mathrm{Tg}$ mice or Foxp $3^{\mathrm{Cre} /+}$ control females were stimulated with plate-bound $\alpha \mathrm{CD} 3$ and $\alpha \mathrm{CD} 28$ (both at $1 \mu \mathrm{g} / \mathrm{ml}$ ) for 6 hours at $37^{\circ} \mathrm{C}$, followed by staining with Ghost Dye Red 710 (Tonbo Biosciences) as well as antibodies against CD4, FOXP3, and YFP/GFP (catalog 50-6498; eBioscience). An LSRFortessa or LSRFortessa $20 \times$ cell analyzer (BD Biosciences) was used for data collection, and Flowjo software (Tree Star) was used for data analysis.

Generation of mixed BM chimeras. T cell-depleted BM cells isolated from femurs and tibiae of Ly5.1- CD4-Cre R27Tg mice or their WT littermates were mixed at a 1:1 ratio with T cell-depleted BM cells taken from Ly5.1 $1^{+}$WT mice $\left(2.5 \times 10^{6}\right.$ each $)$ and i.v. injected into irradiated (950 cGy) Rag1//- mice. Mice were kept on antibiotic water for 4 weeks and harvested for experiments 8 weeks after injection.

Adoptive $T$ cell transfer colitis. To induce colitis, $4 \times 10^{5} \mathrm{CD}^{+} \mathrm{CD} 25^{-}$ CD45RB hi $\mathrm{T}$ cells from either CD4-Cre R27Tg mice or their WT lit-

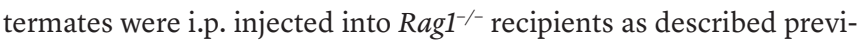
ously (40). To examine Treg-mediated protection from colitis, $4 \times 10^{5}$ $\mathrm{CD} 4^{+} \mathrm{CD} 25^{-} \mathrm{CD} 45 \mathrm{RB}^{\text {hi }} \mathrm{T}$ cells from WT mice were mixed with $2 \times 10^{5}$ $\mathrm{CD} 4{ }^{+} \mathrm{CD} 25^{\mathrm{hi}}$ Tregs from CD4-Cre R27Tg mice or their WT littermates and i.p. injected into $\mathrm{Rag1}^{-/-}$recipients. Mice were sacrificed at the indicated time points or before reaching less than $80 \%$ of the their original BW. The colon was flushed with PBS, and the concentration of IFN- $\gamma$ in the colonic supernatant was measured with an ELISA kit (catalog 43081; BioLegend) according to the manufacturer's instructions. Colonic tissue was saved for histology as well for RNA isolation and quantitative PCR (qPCR). Colonic cells were isolated for flow cytometric analysis as described above.

Histology. To assess immunopathology, different tissues were harvested and immediately fixed in $10 \%$ formalin solution. Paraffinembedded sections were cut (5 $\mathrm{mm}$ thickness) and stained with $\mathrm{H} \& \mathrm{E}$ as described previously (12).

qPCR analysis. For quantification of miR-23 27 24 expression in different thymocyte subsets, double-negative (DN), DP, CD8SP,
FOXPp3 $3^{\text {Thy1.1-CD } 4 S P, ~ a n d ~ F O X P 3 ~} 3^{\text {Thy1.1+ }}$ CD4SP cells from Foxp $3^{\text {Thy1.1 }}$ reporter mice were sorted on a FACSAria II cell sorter (BD Biosciences) with a purity of greater than $95 \%$, followed by RNA isolation using a miRNeasy Kit (QIAGEN). Next, a TaqMan MicroRNA Assay (Thermo Fisher Scientific) was performed as described previously (12). To detect IFN- $\gamma$ levels in the colon, colonic tissues were harvested and RNA was isolated using a TRIzol RNA Kit (Thermo Fisher Scientific). Extracted RNA was converted to cDNA with an iScript cDNA Synthesis Kit (Bio-Rad), followed by qPCR reactions using SYBR Select Master Mix (Thermo Fisher Scientific). All real-time reactions were run on a 7900HT Fast Real-Time PCR System (Thermo Fisher Scientific).

Immunoblotting. FACS-purified naive CD4 T cells were subjected to lysis with RIPA buffer supplemented with $1 \mathrm{mM}$ PMSF for $20 \mathrm{~min}-$ utes. Cell debris was removed by centrifugation at $18,000 \mathrm{~g}$ for 15 minutes at $4^{\circ} \mathrm{C}$, and the supernatants were transferred into fresh tubes. Cell lysates were separated by SDS-PAGE, followed by PVDF membrane (Bio-Rad Laboratories) transfer. Antibodies against $\beta$-actin (catalog AC-74; Sigma-Aldrich); SMAD2/3 (catalog ab3862; Chemicon); FOXO1 (catalog 2880; Cell Signaling Technology); c-Rel (catalog 14-6111; eBioscience); and RUNX1 (catalog 255019; R\&D Systems) were used to visualize the corresponding proteins. Quantification of proteins was calculated with Image J software (NIH).

Luciferase reporter assay. The 3'-UTR region of Rel or Gzmb was cloned into psiCheck2 (Promega). Plasmids containing mutated miR27-binding sites located on the 3'-UTR of Rel or Gzmb were generated by site-directed mutagenesis (Agilent Technologies). WT or mutated psiCHECK2 constructs were transfected along with control miR (miR-155) or miR-27-expressing pMDH-PGK-EGFP plasmid using FuGENE 6 Transfection Reagent (catalog E2691; Promega) into 293T cells (ATCC). Twenty-four hours after transfection, luciferase activity was detected with a Dual-Luciferase Reporter Assay System (catalog E1960; Promega) as described previously (12).

Gene expression-profiling analysis. $\mathrm{CD}^{+} \mathrm{CD} 25^{\mathrm{hi}}$ or $\mathrm{CD} 4^{+} \mathrm{FOXP} 3^{-} \mathrm{YFP}^{+}$ Tregs from 6-week-old CD4-Cre-driven Tg lines or heterozygous Foxp $3^{\mathrm{Cre} / \mathrm{+}} \mathrm{R} 27 \mathrm{Tg}$ female mice, respectively, were FACS sorted. Poly-A RNA-seq was performed using 3 biological replicates for each cell population, similar to what was described previously (12). Upregulated or downregulated genes were identified with a $1.5 \log _{2}$ fold change in $\mathrm{Tg}$ naive T cells (Gene Expression Omnibus [GEO] GSE75909) or Tregs relative to WT using the median expression across 3 replicates in each condition. Gene expression clustering was performed using Cluster 3.0 and visualized using Java TreeView. GO biological processes associated with each set of up- or downregulated genes were queried, and the top $5 \mathrm{GO}$ categories with the greatest significance were selected. Significance of the GO gene enrichment was calculated by first using a Fisher's exact test to analyze enrichment, and then corrected $P$ values using the Benjamini-Hochberg FDR (58). Scatter plot analysis was performed to measured the differences between Tconvs and Tregs in the presence or absence of miR-27 overexpression. The Pearson correlation coefficient was calculated to determine how the $\log _{2}$ ratios of Tconv $\mathrm{Tg}$ / WT and Treg Tg/WT were related with each other. CDF plot analysis was performed as described previously (12). Briefly, target sites were restricted to perfect seed complementarity between positions 2 and 7 of the corresponding miRs with positive Argonaute-binding peaks in the HITS-CLIP database (38). Empirical cumulative distributions were computed using MATLAB (R2014b) to display the $\log _{2}(\mathrm{miR} \mathrm{Tg} / \mathrm{WT}$ ) against the cumulative frequency of all genes or Treg-associated genes 
(59). RNA-seq data for Tregs from CD4-Cre-driven Tg lines and heterozygous Foxp $3^{\mathrm{Cre} /{ }_{+}} \mathrm{R} 27 \mathrm{Tg}$ females are available from the NCBI's GEO database (GEO GSE89548).

Statistics. An unpaired, 2-tailed Student's $t$ test (or 1-way ANOVA for IFN- $\gamma$ expression in the adoptive $\mathrm{T}$ cell transfer colitis studies) was performed using GraphPad Prism 5 software (GraphPad Software).

Study approval. All animal studies were approved by the IACUC of UCSD as well as in accordance with the NIG guidelines for the care and use of laboratory animals and Animal Research: Reporting of In Vivo Experiments (ARRIVE) guidelines.

\section{Author contributions}

LOC and LFL conceived and designed the project. LOC, CJW, SC, and LLL performed the experiments and acquired data.
LOC, SSH, AAK, and LFL analyzed the data. LOC, DTN, and LFL wrote the manuscript.

\section{Acknowledgments}

This work was supported by NIH grants AI103646, AI108651, and AI123782 (to LFL) and AI108651-A1S1 (to LOC). We thank Ye Zheng and Yuqiong Liang at the Salk Institute for Biological Studies for providing experimental assistance and all members of our laboratory for discussions.

Address correspondence to: Li-Fan Lu, Division of Biological Sciences, University of California, San Diego, 9500 Gilman Drive, NSB Room 5314, La Jolla, California 92093-037, USA. Phone: 858.534.9682; E-mail: lifanlu@ucsd.edu.
1. O'Connell RM, Rao DS, Chaudhuri AA, Baltimore D. Physiological and pathological roles for microRNAs in the immune system. Nat Rev Immunol. 2010;10(2):111-122.

2. Lee HM, Nguyen DT, Lu LF. Progress and challenge of microRNA research in immunity. Front Genet. 2014;5:178.

3. Calin GA, et al. Frequent deletions and down-regulation of micro- RNA genes miR15 and miR16 at 13q14 in chronic lymphocytic leukemia. Proc Nat Acad Sci USA. 2002;99(24):15524-15529.

4. Starczynowski DT, et al. Identification of miR-145 and miR-146a as mediators of the $5 \mathrm{q}$ - syndrome phenotype. Nat Med. 2010;16(1):49-58.

5. Tagawa H, Seto M. A microRNA cluster as a target of genomic amplification in malignant lymphoma. Leukemia. 2005;19(11):2013-2016.

6. Simpson LJ, Ansel KM. MicroRNA regulation of lymphocyte tolerance and autoimmunity. JClin Invest. 2015;125(6):2242-2249.

7. O'Connell RM, et al. MicroRNA-155 promotes autoimmune inflammation by enhancing inflammatory T cell development. Immunity. 2010;33(4):607-619.

8. Xiao C, et al. Lymphoproliferative disease and autoimmunity in mice with increased miR-1792 expression in lymphocytes. Nat Immunol. 2008;9(4):405-414.

9. Ponomarev ED, Veremeyko T, Barteneva N, Krichevsky AM, Weiner HL. MicroRNA-124 promotes microglia quiescence and suppresses EAE by deactivating macrophages via the C/EBP- $\alpha$ PU.1 pathway. Nat Med. 2011;17(1):64-70.

10. Boldin MP, et al. miR-146a is a significant brake on autoimmunity, myeloproliferation, and cancer in mice. J Exp Med. 2011;208(6):1189-1201.

11. Guerau-de-Arellano M, et al. Micro-RNA dysregulation in multiple sclerosis favours pro-inflammatory T-cell-mediated autoimmunity. Brain. 2011;134(Pt 12):3578-3589.

12. Cho S, et al. miR-23 27 24 clusters control effector $\mathrm{T}$ cell differentiation and function. J Exp Med. 2016;213(2):235-249.

13. Chandran PA, et al. The TGF- $\beta$-inducible miR23a cluster attenuates IFN- $\gamma$ levels and antigen-specific cytotoxicity in human $\mathrm{CD} 8^{+} \mathrm{T}$ cells. JLeukoc Biol. 2014;96(4):633-645.

14. Guo YE, Riley KJ, Iwasaki A, Steitz JA. Alternative capture of noncoding RNAs or protein-coding genes by herpesviruses to alter host $\mathrm{T}$ cell function. Mol Cell. 2014;54(1):67-79.

15. Isomura I, et al. c-Rel is required for the development of thymic Foxp3+ CD4 regulatory T cells. JExp Med. 2009;206(13):3001-3014.

16. Ruan $\mathrm{Q}$, et al. Development of Foxp3(+) regulatory t cells is driven by the c-Rel enhanceosome. Immunity. 2009;31(6):932-940.

17. Long M, Park SG, Strickland I, Hayden MS, Ghosh S. Nuclear factor-kappaB modulates regulatory $\mathrm{T}$ cell development by directly regulating expression of Foxp 3 transcription factor. Immunity. 2009;31(6):921-931.

18. Zheng Y, Josefowicz S, Chaudhry A, Peng XP, Forbush K, Rudensky AY. Role of conserved non-coding DNA elements in the Foxp3 gene in regulatory T-cell fate. Nature. 2010;463(7282):808-812.

19. Severin ME, et al. MicroRNAs targeting TGF $\beta$ signalling underlie the regulatory $\mathrm{T}$ cell defect in multiple sclerosis. Brain. 2016;139(Pt 6):1747-1761

20. Lu LF, et al. A single miRNA-mRNA interaction affects the immune response in a contextand cell-type-specific manner. Immunity. 2015;43(1):52-64.

21. Liston A, Lu LF, O'Carroll D, Tarakhovsky A, Rudensky AY. Dicer-dependent microRNA pathway safeguards regulatory $\mathrm{T}$ cell function. J Exp Med.2008;205(9):1993-2004

22. Rubtsov YP, et al. Regulatory T cell-derived interleukin-10 limits inflammation at environmental interfaces. Immunity. 2008;28(4):546-558.

23. Guttilla IK, White BA. Coordinate regulation of FOXO1 by miR-27a, miR-96, and miR-182 in breast cancer cells. J Biol Chem . 2009;284(35):23204-23216.

24. Rogler CE, et al. MicroRNA-23b cluster microRNAs regulate transforming growth factor-beta/ bone morphogenetic protein signaling and liver stem cell differentiation by targeting Smads. Hepatology. 2009;50(2):575-584.

25. Tang $\mathrm{W}$, et al. miR-27a regulates endothelial differentiation of breast cancer stem like cells. Oncogene. 2014;33(20):2629-2638.

26. Ma Y, et al. Functional screen reveals essential roles of miR-27a/24 in differentiation of embryonic stem cells. ЕMBO J. 2015;34(3):361-378.

27. Ouyang W, Beckett O, Ma Q, Paik JH, DePinho RA, Li MO. Foxo proteins cooperatively control the differentiation of Foxp3+ regulatory T cells.
Nat Immunol.2010;11(7):618-627.

28. Kerdiles YM, et al. Foxo transcription factors control regulatory $\mathrm{T}$ cell development and function. Immunity. 2010;33(6):890-904.

29. Ouyang W, et al. Novel Foxo1-dependent transcriptional programs control $\mathrm{T}(\mathrm{reg})$ cell function Nature. 2012;491(7425):554-559.

30. Rudra D, Egawa T, Chong MM, Treuting P, Littman DR, Rudensky AY. Runx-CBFbeta complexes control expression of the transcription factor Foxp3 in regulatory T cells. Nat Immunol. 2009;10(11):1170-1177.

31. Ono M, et al. Foxp3 controls regulatory T-cell function by interacting with AML1/Runx1. Nature. 2007;446(7136):685-689.

32. Tone Y, Furuuchi K, Kojima Y, Tykocinski ML, Greene MI, Tone M. Smad3 and NFAT cooperate to induce Foxp3 expression through its enhancer. Nat Immunol. 2008;9(2):194-202.

33. Kitoh A, et al. Indispensable role of the Runx1-Cbfbeta transcription complex for in vivo-suppressive function of FoxP3+ regulatory $\mathrm{T}$ cells. Immunity. 2009;31(4):609-620.

34. Takimoto T, et al. Smad2 and Smad3 are redundantly essential for the TGF-beta-mediated regulation of regulatory $\mathrm{T}$ plasticity and Th1 development. JImmunol. 2010;185(2):842-855.

35. Egawa T, Tillman RE, Naoe Y, Taniuchi I, Littman DR. The role of the Runx transcription factors in thymocyte differentiation and in homeostasis of naive T cells. JExp Med. 2007;204(8):1945-1957.

36. Fassett MS, Jiang W, D'Alise AM, Mathis D, Benoist C. Nuclear receptor Nr4a1 modulates both regulatory T-cell (Treg) differentiation and clonal deletion. Proc Natl Acad Sci U S A. 2012;109(10):3891-3896.

37. Sekiya T, et al. Nr4a receptors are essential for thymic regulatory $\mathrm{T}$ cell development and immune homeostasis. Nat Immunol. 2013;14(3):230-237.

38. Loeb GB, et al. Transcriptome-wide miR-155 binding map reveals widespread noncanonical microRNA targeting. Mol Cell. 2012;48(5):760-770.

39. Bunting K, et al. Genome-wide analysis of gene expression in $\mathrm{T}$ cells to identify targets of the NF-kappa B transcription factor c-Rel. J Immunol. 2007;178(11):7097-7109.

40. Mottet C, Uhlig HH, Powrie F. Cutting edge: cure of colitis by $\mathrm{CD} 4+\mathrm{CD} 25+$ regulatory $\mathrm{T}$ cells. 
J Immunol. 2003;170(8):3939-3943.

41. Gondek DC, Lu LF, Quezada SA, Sakaguchi S, Noelle RJ. Cutting edge: contact-mediated suppression by $\mathrm{CD} 4+\mathrm{CD} 25+$ regulatory cells involves a granzyme B-dependent, perforinindependent mechanism. J Immunol. 2005;174(4):1783-1786.

42. Cao X, et al. Granzyme B and perforin are important for regulatory $\mathrm{T}$ cell-mediated suppression of tumor clearance. Immunity. 2007;27(4):635-646.

43. Xie N, et al. miR-27a regulates inflammatory response of macrophages by targeting IL-10. J Immunol. 2014;193(1):327-334.

44. Wang J, Chen J, Sen S. MicroRNA as biomarkers and diagnostics. J Cell Physiol. 2016;231(1):25-30.

45. Liston A, Linterman M, Lu LF. MicroRNA in the adaptive immune system, in sickness and in health. J Clin Immunol. 2010;30 (3):339-346.

46. Codocedo JF, Ríos JA, Godoy JA, Inestrosa NC. Are microRNAs the molecular link between metabolic syndrome and Alzheimer's disease? Mol Neurobiol. 2016;53(4):2320-2338.
47. Kamal MA, Mushtaq G, Greig NH. Current update on synopsis of miRNA dysregulation in neurological disorders. CNS Neurol Disord Drug Targets. 2015;14(4):492-501.

48. Pua HH, Ansel KM. MicroRNA regulation of allergic inflammation and asthma. Curr Opin Immunol. 2015;36:101-108.

49. Ebert MS, Sharp PA. Roles for microRNAs in conferring robustness to biological processes. Cell. 2012;149(3):515-524.

50. Wohlfert EA, et al. GATA3 controls Foxp $3^{+}$regulatory $\mathrm{T}$ cell fate during inflammation in mice. J Clin Invest. 2011;121(11):4503-4515.

51. Wang Y, Su MA, Wan YY. An essential role of the transcription factor GATA-3 for the function of regulatory T cells. Immunity. 2011;35(3):337-348

52. Rudra D, et al. Transcription factor Foxp 3 and its protein partners form a complex regulatory network. Nat Immunol. 2012;13(10):1010-1019.

53. Koch MA, Tucker-Heard G, Perdue NR, Killebrew JR, Urdahl KB, Campbell DJ. The transcription factor $\mathrm{T}$-bet controls regulatory $\mathrm{T}$ cell homeostasis and function during type 1 inflammation. Nat Immunol. 2009;10(6):595-602.

54. Lu LF, et al. Function of miR-146a in controlling Treg cell-mediated regulation of Th1 responses. Cell. 2010;142(6):914-929.

55. Oldenhove G, et al. Decrease of Foxp $3^{+}$Treg cell number and acquisition of effector cell phenotype during lethal infection. Immunity. 2009;31(5):772-786.

56. Guo Q, et al. Forced miR-146a expression causes autoimmune lymphoproliferative syndrome in mice via downregulation of Fas in germinal center B cells. Blood. 2013;121(24):4875-4883.

57. Liston A, et al. Differentiation of regulatory Foxp $3^{+} \mathrm{T}$ cells in the thymic cortex. Proc Natl Acad Sci USA. 2008;105(33):11903-11908.

58. Benjamini Y, Hochberg Y. Controlling the false discovery rate - a practical and powerful approach to multiple testing. JR Stat Soc Series B Stat Methodol. 1995;57:289-300.

59. Stubbington MJ, et al. An atlas of mouse CD4(+) T cell transcriptomes. Biol Direct. 2015;10:14. 